\title{
MicroRNA-4530 promotes angiogenesis by targeting VASH1 in breast carcinoma cells
}

\author{
TAO ZHANG，LI JING，HONG LI，LINCHAO DING，DONGDONG AI，JIANXIN LYU and LIANJIN ZHONG
}

\begin{abstract}
Key Laboratory of Laboratory Medicine, Ministry of Education, Zhejiang Provincial Key Laboratory of Medical Genetics, College of Laboratory Medicine and Life Sciences, Wenzhou Medical University, Wenzhou, Zhejiang 325035, P.R. China
\end{abstract}

Received November 14, 2015; Accepted March 9, 2017

DOI: $10.3892 / 01.2017 .6102$

\begin{abstract}
The results of our previous study revealed that microRNA (miRNA/miR)-4530 was upregulated in the serum of patients with diabetic retinopathy. The TargetScan miRNA database was used to identify potential targets of miR-4530 and vasohibin-1 (VASH1) was predicted as one of the targets. The results of our previous study demonstrated that miR-4530 was able to promote angiogenesis in human umbilical vein endothelial cells. Therefore, suppressing miR-4530 may be a potentially novel approach towards inhibiting tumor angiogenesis. The present study aimed to investigate the function of miR-4530 and determine whether miR-4530 was able to regulate angiogenesis in breast carcinoma cells by targeting VASH1. MDA-MB-231 and MCF-7 cells were transfected with miR-4530 precursor, anti-miR-4530 and empty vector plasmids. The expression levels of miRNA and mRNA were detected using the reverse transcription-quantitative polymerase chain reaction (RT-qPCR). The expression levels of protein were detected using western blotting. Dual-luciferase reporter assays were used to identify the target of miR-4530. Furthermore, cell proliferation, cell cycle, apoptosis and tube formation assays were used to investigate the function of miR-4530 in vitro. Nude mice were used in a subcutaneous tumor model in vivo study. The results of the present study demonstrated that miR-4530 significantly suppressed proliferation and promoted apoptosis of breast carcinoma cells. In addition, miR-4530 expression promoted angiogenesis in vitro. Results from the western blotting and RT-qPCR revealed that VASH1 was significantly downregulated by miR-4530 in breast carcinoma cells. The results of the present study suggest that miR-4530 promotes
\end{abstract}

Correspondence to: Professor Lianjin Zhong, Key Laboratory of Laboratory Medicine, Ministry of Education, Zhejiang Provincial Key Laboratory of Medical Genetics, College of Laboratory Medicine and Life Sciences, Wenzhou Medical University, Zhongxing North Road, Gaojiaoyuanqu, Cha shan, Wenzhou, Zhejiang 325035, P.R. China

E-mail: zhonglianjin@126.com

Key words: microRNA, breast carcinoma, angiogenesis, vasohibin-1 angiogenesis, inhibits proliferation and induces apoptosis in breast carcinoma cells by suppressing the expression of VASH1.

\section{Introduction}

MicroRNAs (miRs/miRNAs) are short non-coding RNAs that inhibit specific mRNAs by binding to 3'-untranslated region (3'-UTRs) of the target mRNA. Depending on the targets they regulate, they may act as oncogenes or tumor suppressor genes in various types of cancer (1). There has been increasing focus on investigating the role of miRNA in the development of cancer (2-4). The suppressive functions of miRNAs on mRNA translation make them useful as small molecular drugs for gene therapy (5). There has been increasing evidence demonstrating that miRNAs serve essential roles in tumorigenesis (6-8). Furthermore, they may function as oncogenes or tumor suppressor genes.

miR-4530 is located on chromosome 19. In our previous study, the results indicated that miR-4530 was downregulated in the serum of patients with diabetic retinopathy (9). Furthermore, it was demonstrated that miR-4530 was able to promote angiogenesis in endothelial cells. However, the molecular mechanism of miR-4530 in the regulation of angiogenesis remains unclear. In the present study, the role of miR-4530 in the regulation of angiogenesis in breast carcinoma was investigated.

TargetScan was used to predict targets of human miR-4530, and vasohibin-1 (VASH1) was identified in the present study. VASH1 was first reported and named in 2004 by Watanabe et al (10). Its expression was demonstrated to be enhanced in endothelial cells (ECs) during angiogenesis and it inhibited angiogenesis in secreting VASH1 as part of a negative feedback (11).

In the present study, VASH1 was identified to be one of the targets of miR-4530 and may be downregulated by miR-4530. Furthermore, miR-4530 promoted the tube formation of HUVECs and breast carcinoma angiogenesis. Finally, the cellular function experiments demonstrated that miR-4530 suppresses breast carcinoma by affecting MCF-7 and MDA-MB-231 cell proliferation and also induces apoptosis.

\section{Materials and methods}

Cell culture. Human breast carcinoma MDA-MB-231 and MCF-7 cell lines were purchased from the Institute of 
Table I. Primers for quantitative polymerase chain reaction.

\begin{tabular}{ll}
\hline Primer & \multicolumn{1}{c}{ Sequence, 5' $\rightarrow$ ' $^{\prime}$} \\
\hline $\begin{array}{l}\text { miR universal } \\
\text { reverse primer } \\
\text { miR-4530 }\end{array}$ & TGCTGTCAACGATACGCTACG \\
forward primer & ATCAGGACGGGAGCGAAAA \\
GAPDH forward primer & GGAGTCCACTGGCGTCTT \\
GAPDH reverse primer & ATCTTGAGGCTGTTGTCATAC \\
VASH1 forward primer & AGATCCCCATACCGAGTGTG \\
VASH1 reverse primer & GCTTCCAGGCATTTGATTGGC \\
\hline
\end{tabular}

miR, microRNA; VASH1, vasohibin-1.

Biochemistry and Cell Biology, Chinese Academy of Sciences (Shanghai, China). Cells were cultured in Dulbecco's modified Eagle's medium (DMEM; Invitrogen; Thermo Fisher Scientific, Inc., Waltham, MA, USA) containing $10 \%$ fetal bovine serum (FBS; Sigma-Aldrich; Merck KGaA, Darmstadt, Germany) and $100 \mu \mathrm{g} / \mathrm{ml}$ each of penicillin/streptomycin. Human umbilical vein endothelial cells (HUVECs) and HEK-293T cells were purchased from the Institute of Biochemistry and Cell Biology, Chinese Academy of Sciences cultured in RPMI-1640 medium (Invitrogen; Thermo Fisher Scientific, Inc.) containing $10 \% \mathrm{FBS}$ and $100 \mu \mathrm{g} / \mathrm{ml}$ penicillin/streptomycin. Cells were maintained at $37^{\circ} \mathrm{C}$ in at atmosphere containing $5 \% \mathrm{CO}_{2}$ and saturated humidity.

Construction of plasmids and stable transfected cell lines. The plasmids $\mathrm{pPG} / \mathrm{miR} / \mathrm{EGFP}$, pPG-miR4530-EGFP and pPG-miR4530sponge-EGFP were purchased from Shanghai GenePharma Co., Ltd. (Shanghai, China) and were transfected into tumor cells using Lipofectamine 3000 (Invitrogen; Thermo Fisher Scientific, Inc.) according to the manufacturer's protocol. Blasticidin (Sigma-Aldrich; Merck KGaA) was used to screen stable cell lines. miRNA-4530 mimics, inhibitors and their negative control (nc)-mimics, and -inhibitors were purchased from Shanghai GenePharma Co., Ltd.

Reverse transcription-quantitative polymerase chain reaction ( $R T-q P C R)$. TRIzol reagent (Invitrogen; Thermo Fisher Scientific, Inc.) was used to extract total RNA from cells, according to the manufacturer's protocol. A total of $1,000 \mu \mathrm{g}$ RNA was reverse-transcribed into miRNA-cDNA using All-in-One miRNA First-Strand cDNA Synthesis kit (GeneCopoeia, Inc., Rockville, MD, USA) according to the manufacturer's protocol. A total of 1,000 $\mu \mathrm{g}$ RNA was reverse-transcribed into mRNA-cDNA using PrimeScript RT Reagent kit with gDNA Eraser (Takara, Dalian, China) according to the manufacturer's protocol. qPCR was performed to evaluate the expression levels of miRNAs and mRNA using a SYBR Green PCR kit (GeneCopoeia, Inc. Rockville, MD, USA) with the Applied Biosystems StepOnePlus ${ }^{\mathrm{TM}}$ Real-Time PCR system (Thermo Fisher Scientific, Inc., USA). Human U6 was used as an internal control for measuring miRNA expression and GAPDH was used as an internal control for measuring mRNA expression. The expression levels were calculated using
Table II. Thermocycling program for quantitative polymerase chain reaction.

\begin{tabular}{lcc}
\hline Step & Temperature, ${ }^{\circ} \mathrm{C}$ & Duration \\
\hline 1 & 95 & $10 \mathrm{~min}$ \\
2 & 95 & $10 \mathrm{sec}$ \\
3 & 55 & $15 \mathrm{sec}$ \\
4 & 72 & $30 \mathrm{sec}$ \\
Steps 2-4 & - & $40 \mathrm{cycles}$ \\
Melt curve program & - & $60 \mathrm{~min}$ \\
\hline
\end{tabular}

the $2^{-\Delta \Delta C q}$ method (12). The primers for U6 were provided by GeneCopoeia, Inc. All primers are detailed in Table I and the thermocycling conditions are presented in Table II).

Western blotting. Total protein was extracted using radioimmunoprecipitation buffer containing phenylmethylsulfonyl fluoride. Lysates were centrifuged at $14,000 \mathrm{x}$ g for $10 \mathrm{~min}$ at $4^{\circ} \mathrm{C}$ to remove cellular debris and the protein concentration was determined using bicinchoninic acid protein assay kit (Beyotime Institute of Biotechnology, Haimen, China) according to the manufacturer's protocol. A total of $30 \mu \mathrm{g}$ protein was separated on by SDS-PAGE (10\% gel) and transferred onto polyvinylidene fluoride membranes (EMD Millipore, Billerica, MA, USA). Following blocking with milk $2 \mathrm{~h}$ at room temperature, primary antibodies directed against VASH1 (1:1,000 diluted in Beyotime Primary Antibody Dilution Buffer; \#ab199732; Abcam, Cambridge, UK) and GAPDH (1:1,000 diluted in Beyotime Primary Antibody Dilution Buffer; \#2118S; Cell Signaling Technology, Inc., Danvers, MA, USA) were incubated with the membranes overnight at $4^{\circ} \mathrm{C}$. Subsequently, horseradish peroxidase-conjugated secondary antibodies (dilution, 1:10,000 in TBST; \#A0208; Beyotime Institute of Biotechnology, Haimen, China) were incubated with membranes for $2 \mathrm{~h}$ prior to washing the membranes for $1 \mathrm{~h}$ Band signals were detected using enhanced chemiluminescence (Beyotime Institute of Biotechnology), according to the manufacturer's protocol. Western blots were performed $\geq 3$ times.

Luciferase reporter assay. Firstly, TargetScan was used to predict the potential targets of miR-4530. In TargetScan Release 6.2 database, the human species was selected and the microRNA name was entered as 'has-miR-4530'. The request was submitted and the top potential targets were chosen. The gene function and the data of preliminary luciferase reporter assay were evaluated and VASH1 was chosen for further investigation. Human VASH1 mRNA 3'UTR and a mutation of the miR-4530 seed sequence were amplified using PCR with plasmid templates (Shanghai GenePharma Co., Ltd.) and inserted into pmiR-report plasmids. The sequences were subsequently confirmed. A total of $25 \mathrm{ng}$ luciferase reporter plasmids and miR-4530 or miR-negative control (NC) mimics were co-transfected into HEK-293T cells in 24-well plates using Lipofectamine 3000 with RPMI-1640 medium (Invitrogen; Thermo Fisher Scientific, Inc.). After $24 \mathrm{~h}$ of transfection and incubation at $37^{\circ} \mathrm{C}$, the cells were harvested using the passive 
A

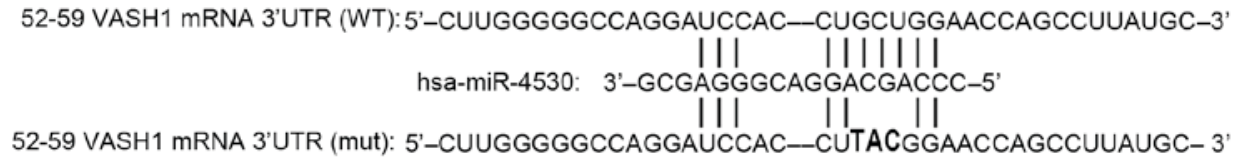

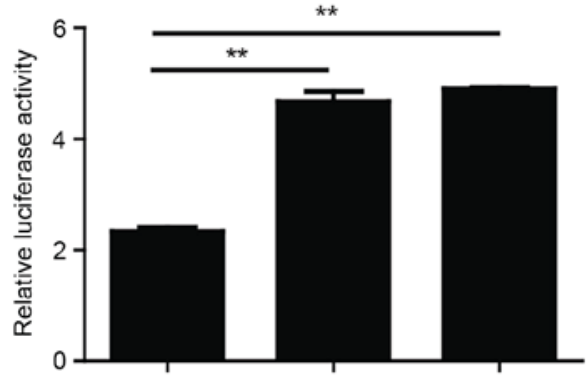

miR-ctrl miR-4530 VASH1-WT VASH1-mut
D

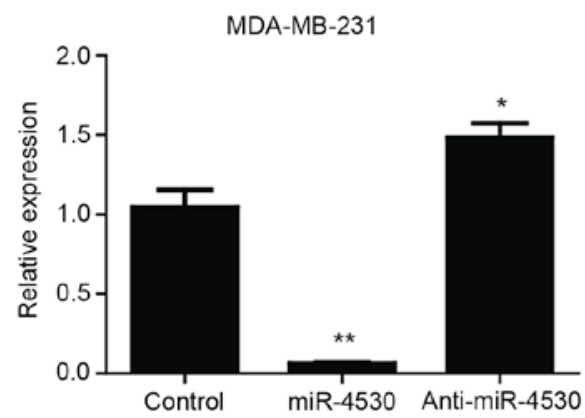

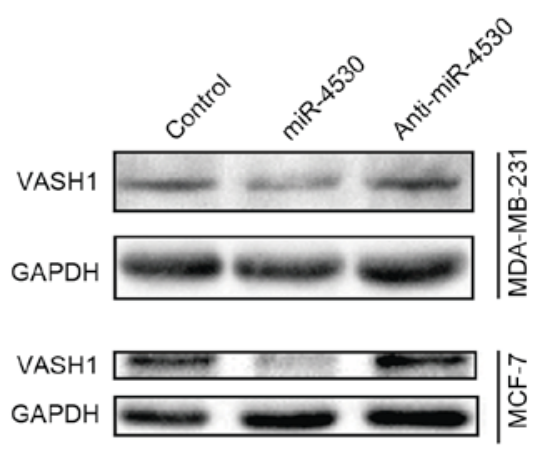

MCF-7

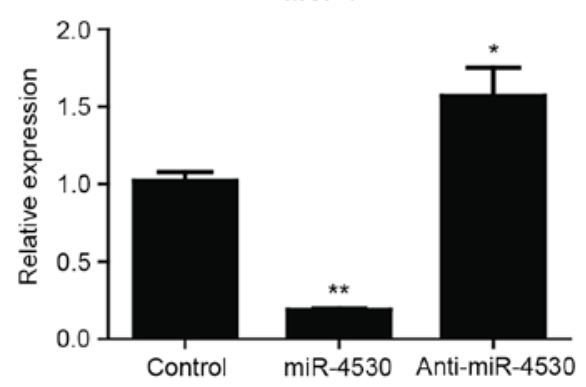

Figure 1. miR-4530 downregulates VASH1 expression in human breast carcinoma cells. (A) WT and Mut VASH1 mRNA of miR-4530 targeting sequences. Mutated sequences are indicated in bold type. (B) miR-4530 mimics inhibited WT but not Mut VASH1 reporter activity in HEK-293T cells. (C) miR-4530 downregulated the expression of endogenous VASH1 protein in human breast carcinoma cells compared with the empty plasmid control group. (D) miR-4530 significantly downregulated the expression of endogenous VASH1 mRNA in human breast carcinoma cells compared with the empty plasmid control group. WT, wild-type; Mut, mutant; 3'UTR, 3'-untranslated region; VASH1, vasohibin-1; miR, microRNA; ctrl, control. ${ }^{*} \mathrm{P}<0.05$, ${ }^{* *} \mathrm{P}<0.01$.

lysis buffer from the Dual-Luciferase Reporter Assay System (Promega Corporation, Madison, WI, USA). The luciferase signal was measured using a Tecan M1000 microplate reader (Thermo Fisher Scientific, Inc. The Renilla luciferase signal was used as an internal control and the firefly luciferase signal corresponded to the expression of firefly luciferase.

Colony formation assays. The cells were counted and seeded into a 6-well plate at a density of 500 cells/well. Cells were cultured for 10 days and medium was replaced with fresh DMEM every 2 days. Subsequently, cells were washed twice with PBS and fixed with $4 \%$ paraformaldehyde for $15 \mathrm{~min}$ at room temperature. Finally, cells were stained with $0.1 \%$ crystal violet (Beyotime Institute of Biotechnology) for $15 \mathrm{~min}$ at room temperature and washed with double-distilled water. The colony formation assay was performed in triplicate and images were captured using a digital camera.

Cell proliferation assays. A total of $3.5 \times 10^{3}$ stable transfected cells were seeded into 96 -well plates and the medium was replaced with fresh DMEM every 2 days. After 24, 48, 72 and $96 \mathrm{~h}$ of incubation, cell proliferation was detected using a Cell Counting kit-8 (CCK-8; Dojindo Molecular Technologies,
Inc., Kumamoto, Japan). At each time point, the medium was replaced with fresh DMEM and the cells were incubated for $1 \mathrm{~h}$ with $10 \mu \mathrm{l}$ CCK-8 solution. Subsequently, all plates were scanned at $450 \mathrm{~nm}$ using a microplate reader. Each experiment was performed independently three times.

Cell cycle and cell apoptosis analysis. For the cell cycle assay, stably transfected cells were collected by centrifugation after $70-80 \%$ confluency was achieved and fixed in $70 \%$ ethanol at $4^{\circ} \mathrm{C}$ overnight. The MDA-MB-231 cells were washed twice with $4^{\circ} \mathrm{C} 1 \mathrm{X}$ PBS and then resuspended in $160 \mu 10.5 \mathrm{mg} / \mathrm{ml}$ RNase A (Nanjing KeyGen Biotech Co., Ltd., Nanjing, China) at $37^{\circ} \mathrm{C}$ for $30 \mathrm{~min}$. Cells were subsequently stained with $50 \mu \mathrm{mol} / 1$ propidium iodide (KeyGen Biotech Co., Ltd.) in the dark for $1 \mathrm{~h}$ at $4^{\circ} \mathrm{C}$ and analyzed using flow cytometry (BD Biosciences, Franklin Lakes, NJ, USA). For analysis of cell apoptosis, stably transfected cells were collected by centrifugation at 2,500 $\mathrm{x}$ g for $5 \mathrm{~min}$ at $4^{\circ} \mathrm{C}$, washed and stained using the annexin $\mathrm{V}$-allophycocyanin (APC)/propidium iodide kit (KeyGen Biotech Co., Ltd.) according to the manufacturer's protocol. The cells were analyzed immediately using flow cytometry (BD Biosciences). Data were analyzed using BD Accuri $^{\text {TM }}$ C6 software (BD Biosciences). 
Tube formation assay. HUVECs and stably transfected MCF-7 cells growing in 6-well plates were serum-starved in RPMI-1640 medium for $24 \mathrm{~h}$ at $37^{\circ} \mathrm{C}$. Frozen $\left(-20^{\circ} \mathrm{C}\right)$ Growth factor-reduced Matrigel (BD Biosciences) was melted in $4^{\circ} \mathrm{C}$ overnight; subsequently, $50 \mu 1$ Matrigel was spread across the bottom of the wells of 96 -well plates. Following solidification of Matrigel at $37^{\circ} \mathrm{C}$, the supernatant medium of the MCF-7 cells was centrifuged $\left(10,000 \mathrm{x}\right.$ g for $5 \mathrm{~min}$ at $\left.4^{\circ} \mathrm{C}\right)$ and collected as tumor cell-conditioned medium (TCM). The supernatant of the HUVECs was discarded and the cells were suspended in TCM. A total of $8 \times 10^{4} /$ well HUVECs were seeded on the Matrigel-coated wells in $100 \mu \mathrm{TMC}$ and incubated at $37^{\circ} \mathrm{C}$ for $10 \mathrm{~h}$. The angiogenic activity was evaluated as the number of tubular structures in each well.

In vivo growth assay. Nude mice were purchased from Shanghai SLAC Laboratory Animal Co., Ltd. (Shanghai, China) and raised in the Laboratory Animal Center of Wenzhou Medical University (Wenzhou, China). In total 18 female nude mice (weight, 20-25 g) were kept in light/dark cycles with sufficient food and water at $20-26^{\circ} \mathrm{C}$ with a humidity of $40-70 \%$ in a specific pathogen-free laboratory animal room. A total of $1 \times 10^{7}$ stably transfected MDA-MB-231 cells were subcutaneously injected into the foreleg armpit of 2-month-old female nude mice ( $n=6 /$ group). Tumor size was measured using a Vernier caliper every 5 days. After 10 weeks, all mice were sacrificed by cervical dislocation and dissected. Tumor tissues were removed for analysis. Animal experimentation was approved by the Laboratory Animal Ethics Committee of Wenzhou Medical University.

Statistics. SPSS v17.0 software (SPSS, Inc., Chicago, IL, USA) was used to perform statistical analysis. Statistical significance was calculated by using the two-tailed Student's t-test. All experiments were performed $\geq 3$ times and data were expressed as mean \pm standard deviation. $\mathrm{P}<0.05$ was considered to indicate a statistically significant difference.

\section{Results}

miR-4530 downregulates VASH1 expression through post-transcriptional repression. The miRNA database TargetScan was utilized to identify the potential targets and the binding site of miR-4530. TargetScan predicted VASH1 as one of the miR-4530 targets with high complementarity. The binding site was located between bases 52 and 59 of the VASH1 3'-UTR (Fig. 1A). Luciferase reporter assays were performed on HEK-293T cells to confirm the association between miR-4530 and VASH1. The results demonstrated that miR-4530 significantly suppressed the firefly luciferase signal compared with the control group and that this inhibitory effect was significantly reversed when the binding site for miR-4530 was mutated (Fig. 1B).

To verify that the suppression of VASH1 expression by miR-4530 does occur in breast carcinoma cell lines, stably transfected MCF-7 and MDA-MB-231 cell lines were constructed. Stably transfected miR-4530 precursor, anti-miR-4530 and empty plasmid groups were produced for each cell line. The expression of miR-4530 in each group was confirmed using qPCR and western blot analyses. The protein expression of VASH1 in the cells with miR-4530 overexpression was downregulated (Fig. 1C), and the mRNA expression was significantly downregulated (Fig. 1D) compared with the corresponding empty plasmid control groups in the two breast carcinoma cell lines. Furthermore, the protein expression of VASH1 in the anti-miR-4530 groups was upregulated (Fig. 1C), and the mRNA expression was significantly upregulated (Fig. 1D) compared with the corresponding empty plasmid control groups. These results suggest that miR-4530 inhibits VASH1 expression through post-transcriptional repression.

Overexpression of miR-4530 results in the inhibition of cell proliferation, colony formation and induces apoptosis. To investigate the role of miR-4530 in breast carcinoma cells, the cell proliferation rate of stably transfected MDA-MB-231 and MCF-7 cells was determined using CCK-8 and colony formation assays. The results of the CCK- 8 assay demonstrated that the proliferation rate of MDA-MB-231 and MCF-7 cells was significantly repressed from $48 \mathrm{~h}$ onwards following overexpression of miR-4530 compared with the control group (Fig. 2A). Furthermore, the ability of colony formation was significantly decreased following overexpression of miR-4530, and significantly increased following the knockdown of miR-4530 compared with the control groups in the two cell lines (Fig. 2B).

To determine whether cell cycle arrest of cancer cells contributed to the repressive effects of miR-4530 on cell proliferation, the percentage of cells in each stage of the cell cycle were analyzed in stably transfected breast carcinoma cells. The result revealed that the overexpression of miR-4530 cells caused cell cycle arrest in $\mathrm{S}$ phase. The proportion of S-phase MDA-MB-231 cells in the miR-4530 overexpression, control and miR-4530 knockdown groups were $36.83,32.27$ and $30.77 \%$, respectively (Fig. 2C). Furthermore, the differences in the percentage of cells in the $\mathrm{S}$ phase in the overexpression and knockdown groups were significant compared with the control group. These results suggest that the overexpression of miR-4530 inhibits breast carcinoma cell proliferation by inhibiting the $\mathrm{S} / \mathrm{G}_{2}$ transition. In addition, cell apoptosis rates were detected using flow cytometry. Stably transfected MDA-MB-231 cells were stained using the Annexin V-APC/propidium iodide kit. The results demonstrated that miR-4530 expression significantly increased the percentage of apoptotic MDA-MB-231 cells compared with the negative control group (Fig. 2D). This suggests that miR-4530 affects apoptotic signaling pathways.

miR-4530 promotes tube formation of HUVECs and breast carcinoma angiogenesis by negatively regulating VASH1 expression. VASH1 has been identified as an inhibitor of angiogenesis in endothelial cells (11). Therefore, it is plausible that miR-4530 may promote angiogenesis in breast carcinoma cells. To investigate this hypothesis, a tube formation assay was performed to confirm whether miR-4530 promotes angiogenesis in breast carcinoma. The results demonstrated that the TCM from the MCF-7 cells stably transfected with the miR-4530 precursor significantly promoted HUVECs to form tube structures compared with TCM from cells stably transfected with the empty vector (Fig. 3A). 

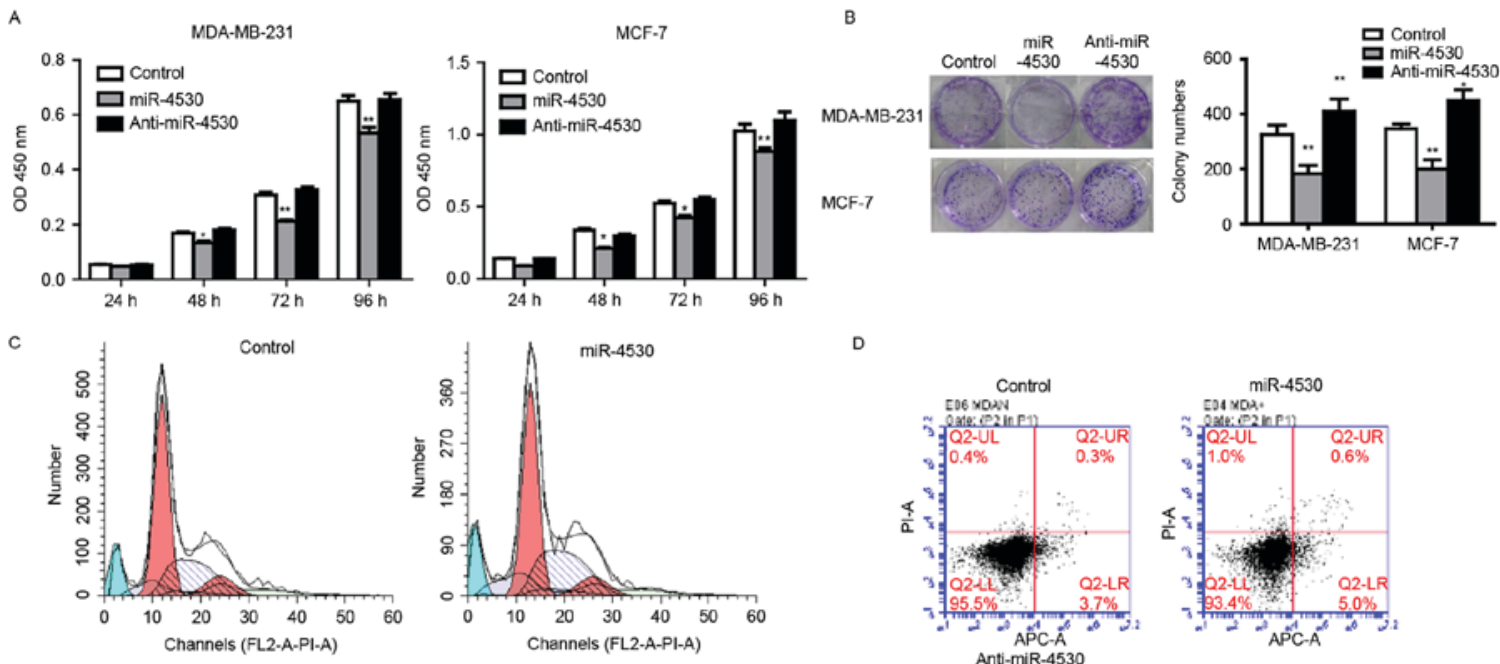

D
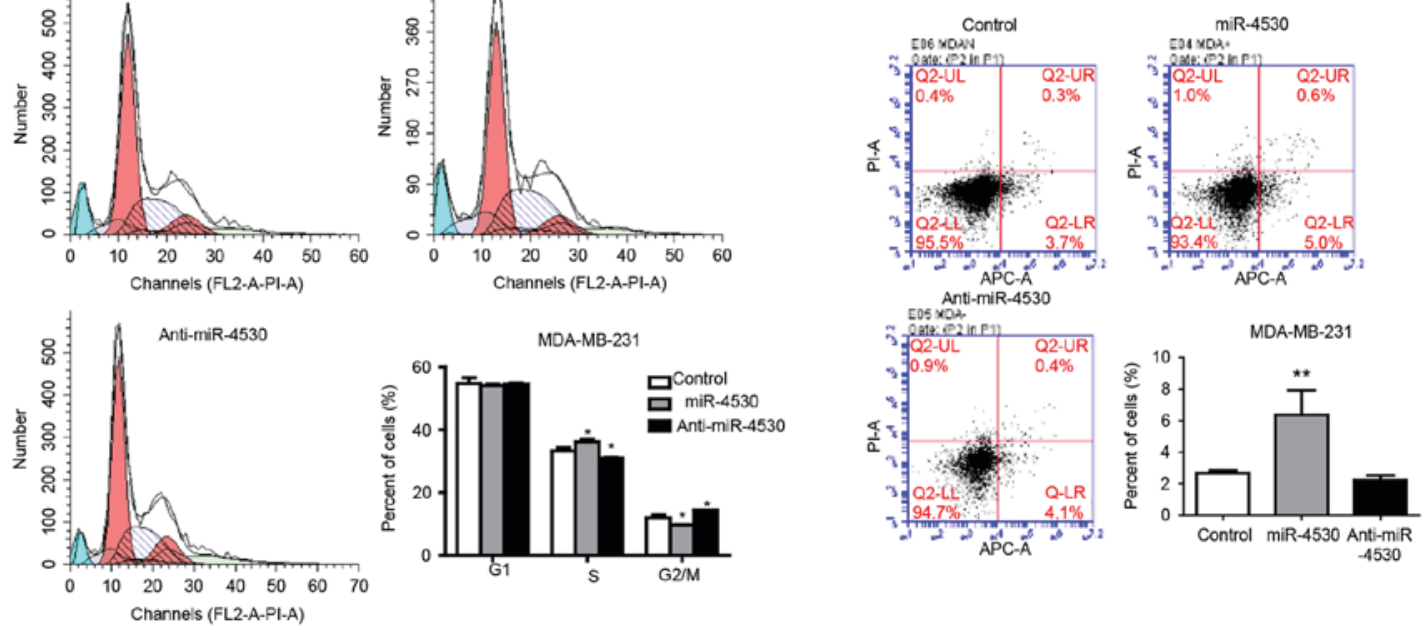

Figure 2. miR-4530 overexpression inhibits breast carcinoma cells growth. (A) Assessment of cell proliferation using a Cell Counting kit-8 assay. The proliferation of MDA-MB-231 and MCF-7 cells was significantly inhibited by overexpression of miR-4530. (B) The ability of colony formation was significantly decreased following overexpression of miR-4530 and increased following the knockdown of miR-4530. (C) The overexpression of miR-4530 in MDA-MB-231 cells inhibited $\mathrm{S} / \mathrm{G}_{2}$ transition. (D) miR-4530 overexpression induces breast carcinoma cell apoptosis in MDA-MB-231 cells. Cell apoptosis was analyzed using fluorescence-activated cell sorting analysis following staining with annexin V-APC/PI kit. miR, microRNA; OD, optical density; APC, allophycocyanin; PI, propidium iodide. ${ }^{*} \mathrm{P}<0.05,{ }^{* *} \mathrm{P}<0.01$.

As expected, the TCM from the anti-miR-4530 group significantly suppressed HUVECs from forming tube structures compared with the control group (Fig. 3A). In addition, tube formation assays were performed on HUVECs transfected with miRNA-4530 mimics, inhibitors or their negative controls. The results were similar to previous results, whereby the mimics significantly increased and the inhibitor significantly decreased the number of tube structures formed compared with the mock-transfected control group (Fig. 3B). It was revealed that miR-4530 mimic-transfected HUVECs exhibited a significant increase in their capability to form tube structures in vitro on Matrigel (Fig. 3B), with a $50 \%$ increase in tube structures compared with miR-4530 NC- or mock-transfected HUVECs. The HUVECs transfected with the miR-4530 inhibitors exhibited significant suppression of angiogenesis in vitro, with a $75 \%$ decrease in tube structures on Matrigel compared with miR-4530 inhibitors NC- or mock-transfected HUVECs (Fig. 3B).

miR-4530 suppresses tumor growth in mice. The nude mice xenograft model was applied to further investigate the effect of miR-4530 on tumorigenicity. MDA-MB-231 cells stably transfected with the miR-4530 precursor, anti-miR-4530 or empty plasmids were subcutaneously injected into 2-month-old female nude mice and the tumor volume was monitored for evaluating the effect of miR-4530 on tumor growth. As presented in Fig. 4A, stable transfection of miR-4530 precursor into MDA-MB-231 cells resulted in a decreased growth rate and significantly decreased tumor size of subcutaneous xenograft tumors by day 60 in nude mice, when compared with the control group (Fig. 4B). Furthermore, cells transfected with anti-miR-4530 exhibited a significant decrease in tumor size by day 80 compared with the control group. The miR-4530 precursor group demonstrated a larger decrease in tumor volume compared with the anti-miR-4530 group. Therefore, cells stably transfected with the miR-4530 precursor exhibited poor survival rates and low tumorigenicity. These results indicate that upregulation of miR-4530 expression is disadvantageous for tumorigenicity.

\section{Discussion}

In our previous study, the results revealed that miR-4530 was upregulated in the serum of patients with diabetic retinopathy (9). Diabetic retinopathy is a complication of diabetes that involves microvessel damage in the retina (13-15). The results of the present study demonstrated that the overexpression and knockdown of miR-4530 regulates angiogenesis. Therefore, this feature may be utilized in suppressing tumor angiogenesis clinically. Angiogenesis serves an essential role in tumor growth and metastasis, and in healthy human development (16). Therefore, in the present study, the effect of miR-4530 on angiogenesis in human breast cancer was investigated.

VASH1 is a novel endogenous angiogenesis inhibitor involved in the negative feedback regulation of 
A

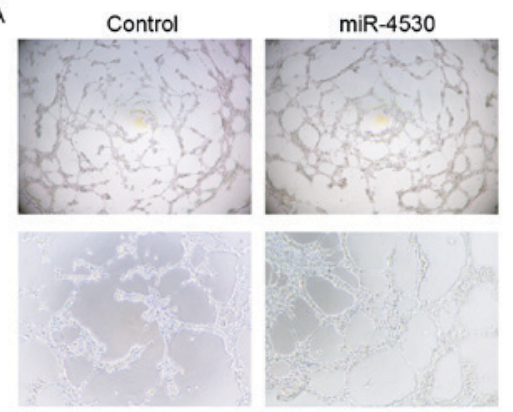

B

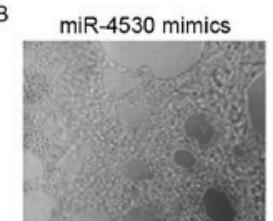

miR-4530 inhibitor

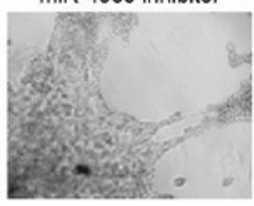

miR-4530 NC

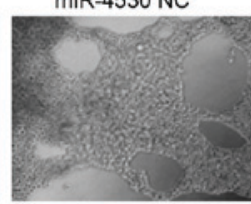

inhibitor NC

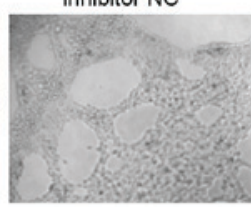

Anti-miR-4530

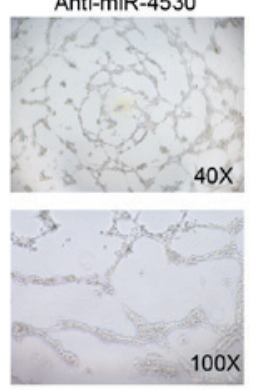

Mock

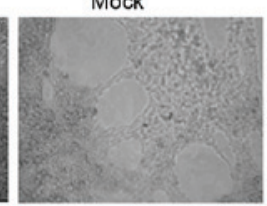

Mock

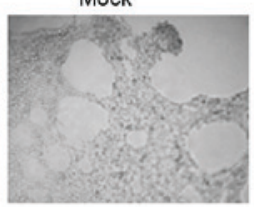

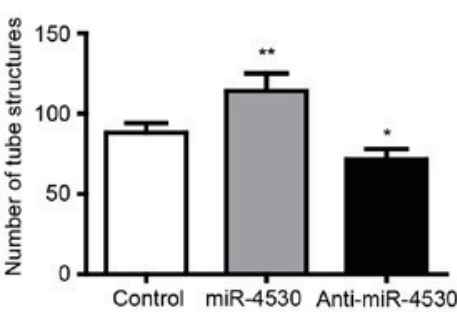
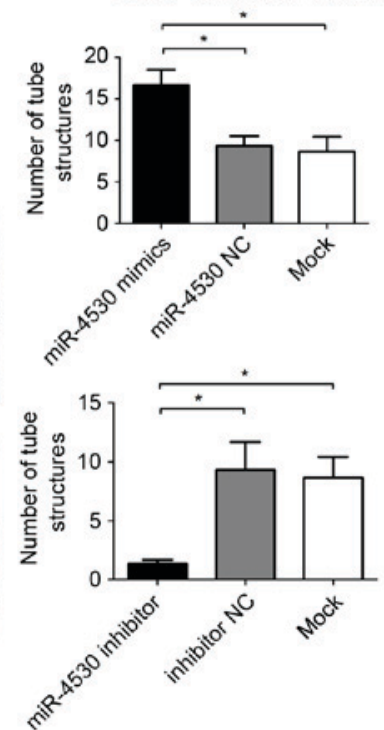

Figure 3. miR-4530 promotes tumor angiogenesis in vitro. (A) HUVECs were cultured in the presence of tumor cell-conditioned medium from MCF-7 cells transfected with miR-4530, anti-miR-4530 or control vector and the number of tubes formed were quantified. (B) The tube formation assay of HUVECs transfected with miRNA-4530 mimics, inhibitors or their negative controls. HUVEC, human umbilical vein endothelial cell; miR, microRNA; NC, negative control. ${ }^{*} \mathrm{P}<0.05,{ }^{* *} \mathrm{P}<0.01$.

A

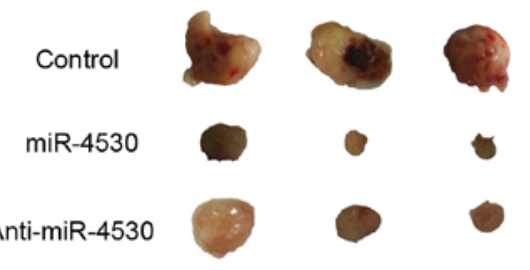

B
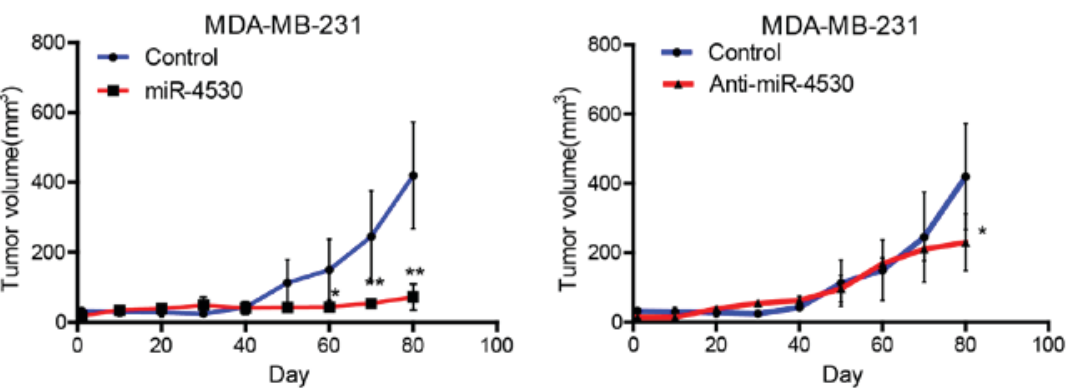

Figure 4. Overexpression of miR-4530 attenuated the growth of MDA-MB-231 cells in vivo. (A) Hypodermic injection of MDA-MB-231 cells into athymic nude mice established subcutaneous xenograft tumors. After 3 months, mice $(n=3)$ from each group were sacrificed. (B) Stable transfection of tumor cells with miR-4530 precursor and anti-miR-4530 resulted in significantly decreased tumor sizes compared with the control group 80 days post-injection. miR, microRNA. ${ }^{*} \mathrm{P}<0.05,{ }^{* *} \mathrm{P}<0.01$.

angiogenesis $(11,17,18)$. It may be a promising candidate for antiangiogenic therapy; however, the molecular mechanism underlying the antiangiogenic activity of VASH1 remains unclear. VASH1 is upregulated in numerous types of tumorous tissue and exhibits therapeutic efficacy in tumor growth (19-22), proliferative retinopathy $(23,24)$ and atherosclerosis $(25)$. Furthermore, VASH1 has been demonstrated to not cause any damage to healthy blood vessels in mice (26), suggesting that 
it is safer and has a limited number of side effects compared with traditional antiangiogenic drugs.

The results of the present study suggest that VASH1 is a direct target gene of miR-4530 in breast carcinoma cells. The overexpression of miR-4530 in breast carcinoma cells resulted in significantly decreased mRNA and protein expression of VASH1 in breast carcinoma cells compared with the control group. Furthermore, it was demonstrated that miR-4530 suppressed breast carcinoma MCF-7 and MDA-MB-231 cell proliferation and induced apoptosis. Miyashita et al (27) reported that the knockdown of VASH1 in ECs induced premature senescence and that those ECs were easily killed by cellular stressors, which may also occur in tumor cells. The knockdown of miR-4530 resulted in an increase in the expression of VASH1 but did not suppress or promote breast carcinoma cell proliferation. These results are consistent with the results of a previous study whereby VASH1 was demonstrated to suppress tumor angiogenesis but not the proliferation of the tumor cells (19). Additionally, the results of the present study suggest that miR-4530 promotes angiogenesis in breast carcinoma. The knockdown of miR-4530 resulted in the suppression of angiogenesis as indicated by the tube formation assays. This may be attributed to the antiangiogenic activity of VASH1.

In vivo assays were also performed in the present study using a xenograft mouse model. The tumor sizes in the miR-4530 overexpression group were significantly decreased with poor survival of cells compared with the control group, which may be attributed to the suppression of cell proliferation and apoptosis. Subcutaneous xenograft tumors were ischemic in the early stages. The majority of tumor cells may have been killed due to starvation. Tumor growth rates in the anti-miR-4530 group appeared to decrease when tumor sizes were $\sim 200 \mathrm{~mm}^{3}$, which may be attributed to a decreased number of microvessels.

VASH1, first identified as an angiogenesis inhibitor in endothelial cells, has now been identified in various other types of cell, including cancer cells (20,28-30). Tamaki et al (22) reported that VASH1 expression in human breast lesions is associated with neovascularization through a compensatory mechanism where antiangiogenesis may negatively regulate intratumoral angiogenesis in human breast cancer. Invasive breast carcinoma or higher nuclear and histological grades of human breast carcinoma exhibited relatively higher VASH1 expression compared with that in ductal carcinoma in situ (31). However, the function and mechanism of VASH1 in cancer cells have rarely been reported.

To the best of our knowledge, the present study is the first to investigate the association between VASH1 and miR-4530, and determine the biological characteristics of VASH1 in human breast carcinoma cells. The results of the present study suggest that VASH1 and miR-4530 are potential therapeutic targets for the treatment of breast cancer particularly with regard to antiangiogenesis therapy.

\section{Acknowledgements}

The present study was supported by the Program for Zhejiang Leading Team of Science and Technology Innovation (grant no. 2010R50048).

\section{References}

1. Shenouda SK and Alahari SK: MicroRNA function in cancer: Oncogene or a tumor suppressor? Cancer Metastasis Rev 28: 369-378, 2009.

2. Jovanovic $\mathrm{M}$ and Hengartner MO: miRNAs and apoptosis: RNAs to die for. Oncogene 25: 6176-6187, 2006.

3. Kent OA and Mendell JT: A small piece in the cancer puzzle: microRNAs as tumor suppressors and oncogenes. Oncogene 25: 6188-6196, 2006.

4. Volinia S, Calin GA, Liu CG, Ambs S, Cimmino A, Petrocca F, Visone R, Iorio M, Roldo C, Ferracin M, et al: A microRNA expression signature of human solid tumors defines cancer gene targets. Proc Natl Acad Sci USA 103: 2257-2261, 2006.

5. Yang $\mathrm{G}$ and Yin B: The advance of application for microRNAs in cancer gene therapy. Biomed Pharmacother 68: 137-142, 2014.

6. Zhou X, Xia Y, Li L and Zhang G: MiR-101 inhibits cell growth and tumorigenesis of Helicobacter pylori related gastric cancer by repression of SOCS2. Cancer Biol Ther 16: 160-169, 2015.

7. Zhang XF, Li KK, Gao L, Li SZ, Chen K, Zhang JB, Wang D, Tu RF, Zhang JX, Tao KX, et al: miR-191 promotes tumorigenesis of human colorectal cancer through targeting C/EBP $\beta$. Oncotarget 6: 4144-4158, 2015.

8. Shen S, Guo X, Yan H, Lu Y, Ji X, Li L, Liang T, Zhou D, Feng XH, Zhao JC, et al: A miR-130a-YAP positive feedback loop promotes organ size and tumorigenesis. Cell Res 25: 997-1012, 2015.

9. Ding L, Ai D, Wu R, Zang T, Jing L, Lu J and Zhong L: Identification of the differential expression of serum microRNA in type 2 diabetes. Biosci Biotechnol Biochem 80: 461-465, 2016.

10. .Watanabe K, Hasegawa Y, Yamashita H, Shimizu K, Ding Y, Abe M, Ohta H, Imagawa K, Hojo K, Maki H, et al: Vasohibin as an endothelium-derived negative feedback regulator of angiogenesis. J Clin Invest 114: 898-907, 2004.

11. Kimura H, Miyashita H, Suzuki Y, Kobayashi M, Watanabe K, Sonoda H, Ohta H, Fujiwara T, Shimosegawa T and Sato Y: Distinctive localization and opposed roles of vasohibin-1 and vasohibin-2 in the regulation of angiogenesis. Blood 113: 4810-4818, 2009

12. Livak KJ and Schmittgen TD: Analysis of relative gene expression data using quantitative PCR and the 2-(Delta DeltaC(T)) method. Methods 25: 402-408, 2001.

13. Giacco F and Brownlee M: Oxidative stress and diabetic complications. Circ Res 107: 1058-1070, 2010.

14. Du Y, Veenstra A, Palczewski K and Kern TS: Photoreceptor cells are major contributors to diabetes-induced oxidative stress and local inflammation in the retina. Proc Natl Acad Sci USA 110: 16586-16591, 2013.

15. Tang J and Kern TS: Inflammation in diabetic retinopathy. Prog Retin Eye Res 30: 343-358, 2011.

16. Hanahan D and Folkman J: Patterns and emerging mechanisms of the angiogenic switch during tumorigenesis. Cell 86: 353-364, 1996.

17. Kerbel RS: Vasohibin: The feedback on a new inhibitor of angiogenesis. J Clin Invest 114: 884-886, 2004.

18. Shimizu K, Watanabe K, Yamashita H, Abe M, Yoshimatsu H, Ohta $\mathrm{H}$, Sonoda $\mathrm{H}$ and Sato Y: Gene regulation of a novel angiogenesis inhibitor, vasohibin, in endothelial cells. Biochem Biophys Res Commun 327: 700-706, 2005.

19. Li D, Zhou K, Wang S, Shi Z and Yang Z: Recombinant adenovirus encoding vasohibin prevents tumor angiogenesis and inhibits tumor growth. Cancer Sci 101: 448-452, 2010.

20. Hosaka T, Kimura H, Heishi T, Suzuki Y, Miyashita H, Ohta H, Sonoda H, Moriya T, Suzuki S, Kondo T and Sato Y: Vasohibin-1 expression in endothelium of tumor blood vessels regulates angiogenesis. Am J Pathol 175: 430-439, 2009.

21. Yoshinaga K, Ito K, Moriya T, Nagase S, Takano T, Niikura H, Yaegashi $\mathrm{N}$ and Sato Y: Expression of vasohibin as a novel endothelium-derived angiogenesis inhibitor in endometrial cancer. Cancer Sci 99: 914-919, 2008.

22. Tamaki K, Moriya T, Sato Y, Nagase S, Takano T, Niikura H, Yaegashi $\mathrm{N}$ and Sato Y: Vasohibin-1 in human breast carcinoma: A potential negative feedback regulator of angiogenesis. Cancer Sci 100: 88-94, 2009.

23. Sato H, Abe T, Wakusawa R, Asai N, Kunikata H, Ohta H, Sonoda H, Sato Y and Nishida K: Vitreous levels of vasohibin-1 and vascular endothelial growth factor in patients with proliferative diabetic retinopathy. Diabetologia 52: 359-361, 2009. 
24. Shen J, Yang X, Xiao WH, Hackett SF, Sato Y and Campochiaro PA: Vasohibin is up-regulated by VEGF in the retina and suppresses VEGF receptor 2 and retinal neovascularization. FASEB J 20 723-725, 2006.

25. Yamashita H, Abe M, Watanabe K, Shimizu K, Moriya T, Sato A, Satomi S, Ohta H, Sonoda H and Sato Y: Vasohibin prevents arterial neointimal formation through angiogenesis inhibition. Biochem Biophys Res Commun 345: 919-925, 2006.

26. Heishi T, Hosaka T, Suzuki Y, Miyashita H, Oike Y, Takahashi T, Nakamura T, Arioka S, Mitsuda Y, Takakura T, et al: Endogenous angiogenesis inhibitor vasohibin1 exhibits broad-spectrum antilymphangiogenic activity and suppresses lymph node metastasis. Am J Pathol 176: 1950-1958, 2010.

27. Miyashita H, Watanabe T, Hayashi H, Suzuki Y, Nakamura T, Ito S, Ono M, Hoshikawa Y, Okada Y, Kondo T and Sato Y: Angiogenesis inhibitor vasohibin-1 enhances stress resistance of endothelial cells via induction of SOD2 and SIRT1. PLoS One 7: e46459, 2012.
28. Sato Y: Is vasohibin-1 for more than angiogenesis inhibition? J Biochem 149: 229-230, 2011.

29. Yan Y, Shen Z, Ye Y, Jiang K, Zhang H, Shen C, Mustonen H, Puolakkainen P and Wang S: A novel molecular marker of prognosis in colorectal cancer: Vasohibin-1. Med Oncol 31: 816, 2014.

30. Liu S, Han B, Zhang Q, Dou J, Wang F, Lin W, Sun Y and Peng G: Vasohibin-1 suppresses colon cancer. Oncotarget 6: 7880-7898, 2015.

31. Tamaki K, Sasano H, Maruo Y, Takahashi Y, Miyashita M, Moriya T, Sato Y, Hirakawa H, Tamaki N, Watanabe M, et al: Vasohibin-1 as a potential predictor of aggressive behavior of ductal carcinoma in situ of the breast. Cancer Sci 101: 1051-1058, 2010 . 